\title{
Islamwissenschaft und relevante Redundanz
}

MAURUS REINKOWSKI (FREIBURG)

\section{Orientalistik und Islamwissenschaft}

Der Orient war nicht die einzige Welt, die außerhalb Europas lag, aber er war schon seit langem das Gegenüber par excellence, das eigentliche Andere. Die akademische Disziplin, die sich seit dem 19. Jahrhundert diesem hauptsächlichen Anderen (nur die beiden Amerikas, Australien und das südliche Afrika waren ausgenommen) widmete, war und ist die Orientalistik. Den Orient erforschende wissenschaftliche Gesellschaften, wie die Société Asiatique (gegr. 1822), Royal Asiatic Society (gegr. 1824), American Oriental Society (gegr. 1842) oder Deutsche Morgenländische Gesellschaft (gegr. 1845), wurden ins Leben gerufen. Bis weit in das 20. Jahrhundert hinein pflegten die Orientalisten einen umfassenden Anspruch: Die Gelehrten sollten die Gesamtheit des Orients zu erfassen und umfassen imstande sein. So wurde 1949 mit Oluf Krückmann (1904-1984) ein Wissenschaftler an das Orientalische Seminar der Universität Freiburg berufen, ,der in seiner Person noch die ganze Welt des Vorderen Orients vereinte: Die Keilschriftsprachen ebenso wie die Ägyptologie und die Islamwissenschaft“. ${ }^{1}$ Carl Heinrich Becker (1876-1933), der die Islamwissenschaft als reguläres Fach in Deutschland mitbegründete, aber bereits im Alter von etwa 40 Jahren in die Wissenschaftspolitik wechselte, interessierte sich ,neben orientalischer Sprache, Literatur, Geschichte und Religion für Archäologie und Kunstgeschichte, für Geschichte der Mathematik, Medizin und Chemie, für Papyrusforschung, für islamisches Recht, für modernen orientalischen Volksaberglauben, für Geschichte Zentralafrikas, für die Kolonial- und Islampolitik der Großmächte, für orientalisches Pressewesen, für die Auseinandersetzung des Orients mit dem vordringen-

1 Horst Steible: „Geschichte des Orientalischen Seminars“, http://www.orient.unifreiburg.de/geschichte.html vom 5. April 2007. - Der Koranforscher Josef Horovitz (1874-1931) wird 1914 auf eine Professur nach Frankfurt/M berufen, deren Stifter als Aufgabengebiete semitische Philologie mit Berücksichtigung der targumischen und talmudischen Literatur sowie Islamkunde festgelegt hatte; Shlomo Dov Fritz Goitein: „Nachruf auf Josef Horovitz“, in: Der Islam 22 (1935), S. 122-127, hier S. 126. 
den Okzident.“² Die Gründe für diese enzyklopädische Ausrichtung lagen zum einen an der damals noch geringeren Fächerdifferenzierung innerhalb der Orientalistik und zum anderen an einer oft der orientalistischen Spezialisierung vorausgehenden Ausbildung in Fächern wie Theologie und klassische Philologie. ${ }^{3}$

Die Islamwissenschaft wurde immer auch als Teil der Orientalistik verstanden, und in der Tat: Die Ähnlichkeiten zwischen diesen beiden sind bemerkenswert - allein schon in der Hinsicht, dass sie weniger Disziplinen ähneln als konglomerierten Wissenschaftstraditionen.

Beide, Islamwissenschaft und Orientalistik, agieren und argumentieren in ihrer Eigenschaft als geisteswissenschaftliche Fächer, bei denen - wie schon in der Einführung zum Sammelband angesprochen wurde - das Krisenbewusstsein ein grundlegendes Merkmal ihrer Selbstbefindlichkeit ist. Klagen gehört zum akademischen Handwerk und zu einer erfolgreichen geisteswissenschaftlichen Wissenschaftspolitik. So bemängelte Hans Robert Roemer (1915-1997), der vielleicht erfolgreichste orientalistische und islamwissenschaftliche Wissenschaftslobbyist der Nachkriegszeit, in den 1970er Jahren die fehlende Ausstattung mit Personal - eine Dekade, die nach heutigem Verständnis als eher noch angenehm gelten muss. ${ }^{4}$ Gemeinsam ist auch Islamwissenschaft und Orientalistik ein schon

2 Hellmut Ritter: „Nachruf auf Carl Heinrich Becker“, in: Der Islam 24 (1937), S. 175-185, hier S. 180. - Siehe als weiteres anschauliches Beispiel wiederum den Nachruf von Fritz Meier auf Hellmut Ritter (1892-1971), in: Der Islam 48 (1972), S. 193-205, hier S. 199f: „Ritters gelehrte hinterlassenschaft ist eine der reichsten und markantesten, die die geschichte seines faches aufzuweisen hat. Sie ist keinesweges spezialisiert, sondern umfasst das arabische, persische, türkische, kurdische und aramäische sprachgebiet, reicht von arbeiten über grammatik, dialektologie, rhetorik über geschichte, wirtschaftsgeschichte, geschichte der naturwissenschaften und technik, magie, literaturgeschichte, handschriftenkunde, filosofie, theologie, mystik, volkskunde, dichtung, theater bis zur musik und ruht auf einer allgemeinbildung, die weit über das gewohnte mass hinausreicht." Siehe auch den Nachruf von Albrecht Noth auf den breit aufgestellten Orientalisten cum Islamwissenschaftler cum promovierten Juristen Otto Spies (1901-1981), in: Der Islam 59 (1982), S. 185188, hier S. 186.

3 Johann W. Fück (1894-1974) unterrichtete zuerst im Schuldienst klassische Philologie und Hebräisch, Nachruf von Werner Ende, in: Der Islam 53 (1976), S. 193; Richard F. Kreutel (1916-1981) hatte eine abgeschlossene Ausbildung als Gymnasiallehrer für Klassische Philologie, Nachruf von Karl Teply, in: Der Islam 59 (1982), S. 190-194, hier S. 190; Rudi Paret (1901-1983), prominenter Koranübersetzer und -interpret, studierte zuerst Evangelische Theologie in Tübingen; siehe Nachruf von Josef van Ess, in: Der Islam 61 (1984), S. 1-7, hier S. 2.

4 ,Mit der Stagnation der Haushalte hat auch schon der Abstieg begonnen, in der Orientalistik noch ehe der ohnehin erst zu Beginn der sechziger Jahre begonnene Ausbau einen Abschluß gefunden und Dauerregelungen an die Stelle von Provisorien und Improvisationen getreten wären.", Eröffnungsansprache des Ersten Vorsitzenden der Deutschen Morgenländischen Gesellschaft, Hans Robert Roemer, zum Orientalistentag 1975 in Freiburg, in: Wolfgang Voigt (Hg.), XIX. Deutscher Orienta- 
nicht mehr ganz neues nagendes Gefühl des Unbehagens angesichts der Zukunft des Faches. 5

Ähnlichkeiten solcher Art dürfen mit Recht trivial genannt werden: Sie könnten auch für viele andere Fächer ausgemacht werden. Aber es gibt noch weit bedeutendere Hinweise auf eine tiefgehende Verwandtschaft von Orientalistik und Islamwissenschaft: Die Orientalistik ist für die Islamwissenschaft nicht nur von Bedeutung, weil diese institutionell noch sehr oft in orientalistischen Arbeitseinheiten angesiedelt ist; die Islamwissenschaft ist in vielen ihrer Eigenschaften noch ein zutiefst orientalistisches Fach. Folgt man den Selbstdarstellungen der Orientalistik oder Islamwissenschaft, so lassen sich in den meisten Selbstbeschreibungen die Begriffe, orientalistisch` und, islamwissenschaftlich ‘ mühelos austauschen. Die Islamwissenschaft scheint so eine Orientalistik im kleineren Format zu sein; sie praktiziert eine ähnliche generell-umfassende Sicht ihres Aufgabenbereichs wie dies die Orientalistik tut. So heißt es in einer Selbstdarstellung des Faches Islamwissenschaft: „Als Kulturwissenschaft auf philologisch-historischer Grundlage beschäftigt sie sich prinzipiell mit allen Kulturen und Gesellschaften, in denen Muslime in Geschichte und Gegenwart, also etwa seit 600 nach Christus, entweder die Mehrheit oder jedenfalls eine aktive Minderheit gestellt haben."6 Beiden, Islamwissenschaft und Orientalistik, ist der Hinweis auf die große Breite des Faches gemein - in Gestalt eines unermesslichen geographischen Raumes und einer enormen Vielfalt von Sprachen (neben den klassischen Sprachen der Islamwissenschaft, Arabisch, Persisch und Türkisch bzw. Osmanisch, können unter anderen Suaheli, Urdu, Dari, Kurdisch, Paschto und Bahasa Melayu/Indonesia genannt werden). ${ }^{7}$

Beiden, Orientalistik und Islamwissenschaft, ist auch gemeinsam, dass trotz dieser Breite und Fülle von Forschungsfeldern und internen Unterdisziplinen ein-

listentag vom 28. September bis 4. Oktober 1975 in Freiburg im Breisgau, Wiesbaden: Steiner 1977, S. XV.

5 So sinniert Franz Rosenthal in seinem Beitrag „Die Krise der Orientalistik“, in: Fritz Steppat (Hg.), XXI. Deutscher Orientalistentag vom 24. bis 29. März 1980 in Berlin. Vorträge, Wiesbaden: Steiner 1983, S. 10-21, hier S. 13: „So müssen wir uns denn fragen, woher das Gefühl des Unbehagens kommt, das wir häufig haben, wenn wir an die Zukunft unseres Studienbereichs denken".

6 Lutz Richter-Bernburg: „Islamwissenschaft“, in: Florian Keisinger/Roland Berger (Hg.), Wozu Geisteswissenschaften? Kontroverse Argumente für eine überfällige Debatte, Frankfurt/M, New York: Campus 2003, S. 124-130, hier S. 126f. - Extrem weit gespannt ist die Aufgabenbeschreibung der Islamwissenschaft in Tübingen, http://www.uni-tuebingen.de/orientsem/islampl.htm\#de vom 14.10.2007.

7 Man vergleiche auch die Darstellung der geographischen und thematischen Weite der Islamwissenschaft auf den Seiten der Freiburger Islamwissenschaft: „Der geographische Rahmen erstreckt sich von der Atlantikküste Nordafrikas über die Arabische Halbinsel, Südosteuropa und die Türkei, Iran, Mittel- und Zentralasien bis nach Indonesien. Der inhaltliche Rahmen umspannt Religion, Geschichte, Sprachen und Literatur ebenso wie Recht, Philosophie, Kunst, Gesellschaft, Wirtschaft und historische Landeskunde.“, http://www.orient.uni-freiburg.de/islam vom 14.10.2007. 
zelne Forschungsfelder hervorstechen. In der Orientalistik sind dies die Sinologie, Indologie und Islamwissenschaft. Im Fall der deutschsprachigen Islamwissenschaft dominieren diejenigen Regionen, die im weitesten Sinne als die Geburtsländer der monotheistischen Religionen gelten können, also der Nahe Osten. Nimmt man die gesamte islamische Welt als Maßstab, so erscheinen Gebiete wie Israel, Palästina und Syrien stark vergrößert, währenddessen bereits Länder wie Iran in dieser Matrix übermäßig klein geraten, ganz zu schweigen von so ,fernen ‘ Gebieten wie Südostasien. Aus dieser Perspektive erscheint - überspitzt ausgedrückt - der Islam im subsaharischen Afrika, in Südasien oder Südostasien als eine Art asiatischer Restislam. ${ }^{8}$

Es kann auch nicht nur Zufall sein, dass der Islamwissenschaft jede eigentliche Standesvertretung fehlt: Weder die, die gesamte Orientalistik umfassende Deutsche Morgenländische Gesellschaft noch die 1993 gegründete und nur auf die Moderne bezogene Deutsche Arbeitsgemeinschaft Vorderer Orient für gegenwartsbezogene Forschung und Dokumentation können als ideale Lobbyisten des islamwissenschaftlichen Fächerverbundes gelten. Oder ist nicht doch die Islamwissenschaft mit ihren - ähnlich wie in der Orientalistik - undefinierten Fachgrenzen am besten in einer orientalistischen Interessenorganisation aufgehoben? Die Frage stellt sich ohnehin: Wer ist eigentlich Islamwissenschaftler bzw. wer wird eigentlich von anderen Islamwissenschaftlern als Islamwissenschaftler angesehen? Eine Umfrage unter deutschen Islamwissenschaftlerinnen und Islamwissenschaftlern würde hier höchst disparate Ergebnisse zeitigen.

Orientalistik und Islamwissenschaft teilen sich nicht nur die enorme geographische Breite und thematische Vielfalt, sondern auch die Betonung der historischen Tiefe: „Es ist das Besondere der Orientalistik - zumindest in der Tradition der Deutschen Morgenländischen Gesellschaft, und dies gilt es auch in jedem Fall zu bewahren -, dass sie sich mit jeweils zeitgenössischen Fragen nur aus einer - eben gerade auch sprachlich-philologisch - fundierten Kenntnis vorausgegangener Epochen heraus auseinanderzusetzen bereit ist. “9 Während das Argument einer historischen Tiefe orientalischer oder islamischer Zivilisationen kaum von der Hand zu weisen ist, so ist die Vorstellung, dass Geschichte und Gegenwart unauflösbar ineinander verschränkt sind und eine institutionalisierte Trennung in moderne und historische Studien weitgehend sinnlos wäre ,in einer Welt, wo uralt verwurzelte Traditionen noch heute wirksam sind“" ${ }^{10}$ doch nicht

8 Zur marginalen Stellung Afrikas und Südasiens im Rahmen der Islamwissenschaft siehe die Beiträge von Roman Loimeier und Jan-Peter Hartung in diesem Band.

9 Eröffnungsansprache des Ersten Vorsitzenden der Deutschen Morgenländischen Gesellschaft, Herrmann Jungraithmayr, auf dem XXV. Orientalistentag 1991 in München, in: Cornelia Wunsch (Hg.), XXV. Deutscher Orientalistentag, vom 8. bis 13.4.1991 in München. Vorträge, Stuttgart: Steiner 1994, S. 1-7, hier S. 5.

10 Eröffnungsansprache des Ersten Vorsitzenden der Deutschen Morgenländischen Gesellschaft auf dem XVII. Orientalistentag 1968 in Würzburg, in: Wolfgang Voigt (Hg.), Deutscher Orientalistentag: Vorträge. Vom 21. bis 27. Juli 1968 in Würzburg, 
ungefährlich: Heutige Gesellschaften werden in Haftung genommen für vergangene Kulturen.

\section{Zwischen philologischer Verankerung und disziplinärer Verunsicherung}

Die Selbstverteidigungstechniken und -argumente der islamwissenschaftlichen und orientalistischen Fachtraditionen sind bis in die unmittelbare Gegenwart ähnlich gewesen. Beiden ist gemein, dass sie jahrzehntelang zwischen der Scylla übermäßiger philologischer Selbstzufriedenheit und der Charybdis disziplinärer Verunsicherung - angesichts der Herausforderung durch methodisch besser verankerte und wissenschaftspolitisch stärker gerüstete Fächer wie Geschichte, Politikwissenschaft und Soziologie - gesegelt sind.

Wenn die große und auch heroische Zeit der Orientalistik das späte 19. und das frühe 20. Jahrhundert war, so lässt sich für die zweite Hälfte des 20. Jahrhundert ein Bedeutungsverfall des Orients festhalten: Der Orient, auch in den Orientwissenschaften, wird nur noch als ein Hilfskonstrukt verstanden, ja sogar als ein höchst problematischer Begriff - auch infolge der schneidenden Kritik, die an den Konzepten des Orients, des Orientalismus und der Orientalistik geäußert wurde.

1978 erschien Edwards Said Orientalism. In diesem Buch, das die Diskussionen der letzten dreißig Jahre geprägt hat, ${ }^{11}$ unternimmt Said seine Beweisführung im Wesentlichen anhand europäischer Reiseliteratur und westlicher orientalistischer Werke. Orientalismus bedeutet ihm dabei Verschiedenes, das dennoch eng ineinander verwoben ist: (a) die akademische Wissenschaft der Orientalistik; (b) eine Denkweise, die den Unterschied zwischen West und Ost zur Voraussetzung ihrer Argumentation macht; (c) ein Medium der Dominanz und Kontrolle über ,den Orient'. Der Orient ist also nicht nur eine Idee, sondern dient als Objekt zur Ausübung realer Macht: Europäischer Kolonialismus und westlicher Imperialismus stützen den Orientalismus und stehen hinter dessen Projektionen. Und natürlich helfen die Projektionen des Orientalismus wiederum die Machtverhältnisse zu zementieren. Der Orientalismus ist demnach eine sehr stabile und zähe Konstruktion, die nicht einfach aufgebrochen werden kann, und die sich durchaus immer wieder an neue Umstände anpassen kann.

Zwar weiß man heute genug über die Schwächen und Einseitigkeiten von Saids Darstellung, ${ }^{12}$ aber trotzdem sind wir in der Debatte über die Orientalistik

Teil 1. 1969, Zeitschrift der Deutschen Morgenländischen Gesellschaft: Supplement; 1, S. XXXI.

11 Der Ruhm von Saids Buch ist gerechtfertigt, aber doch auch einseitig. Bereits 1963 hatte Anouar Abdel-Malek (,L'orientalisme en crise“, Diogène 44/1983, S. 103140) alle wesentlichen Elemente der Orientalismuskritik formuliert.

$12 \mathrm{Zu}$ einer ausgewogenen und rezenten Zusammenfassung der Thesen Saids, seiner Kritiker und Nachfolger siehe Zachary Lockman: Contending Visions of the Middle 
noch kaum über Orientalism hinausgedrungen. ${ }^{13}$ Man wird wohl erst dann von einem wirklichen Fortschritt in der Orientalismuskritik sprechen können, wenn nicht in jedem Beitrag zur orientalistischen und islamwissenschaftlichen Wissenschaftsgeschichte (wie es auch in diesem Band der Fall ist) immer wieder Said erwähnt wird.

In anglo-amerikanischen Ländern wird jedenfalls heute all dasjenige, was sich mit ,dem Orient" beschäftigen will, ihn also als solchen als gegeben annimmt, als Orientalismus verworfen. ${ }^{14}$ Jemand anderen einen ,great Orientalist zu nennen, kann dann nichts anderes mehr als eine gewollte Beleidigung sein. In deutschsprachigen Ländern dagegen gibt es noch zahlreiche ,Orientalische Seminare': Angefangen von Wien, Zürich, und Basel über Freiburg, Tübingen, Frankfurt, Gießen, Bonn, Köln, bis nach Halle und Leipzig. ${ }^{15}$

Das ist also die eine Seite: Wir sehen vor uns eine - angeblich - machtvolle Geschichte der Deutungshoheit über den Orient, ausgeübt von wissenschaftlichen Hohepriestern, die zwar selbst unentrinnbar in ihren Diskursen gefangen sind, die aber auch allen anderen ihre Rede vom Orient aufzwingen können. Aber haben wir - im Gegensatz zur Rede der deutungsmächtigen Islamwissenschaft als einer der letzten großen Bastionen eines wissenschaftlichen Orientalismus - haben wir nicht auf der anderen Seite ganz andere, viel verhaltenere und auch kleinmütigere Töne im Ohr? ${ }^{16}$ Wie steht es eigentlich mit dem Selbstbewusstsein der Islamwissenschaft und der Orientalistik?

East. The History and Politics of Orientalism, Cambridge: Cambridge University Press 2004, Kap. 6: „Said's Orientalism: A Book and its Aftermath“.

13 Bemerkenswert ist die Vermutung von Baber Johansen: „Politics and Scholarship: The Development of Islamic Studies in the Federal Republic of Germany“, in: Tareq Y. Ismael (Hg.), Middle East Studies. International Perspectives on the State of the Art, New York u.a.: Praeger 1990, S. 71-130, hier S. 73, dass der Erfolg von Saids Buch sich seiner einseitigen Denunziation der Orientalistik zu verdanken habe. Entlastet von ihren Schuldgefühlen, hätten alle anderen Teile der westlichen Öffentlichkeit auf den Sündenbock Orientalistik als angeblichen Hauptverantwortlichen für die Entstehung des Orientalismus zeigen können.

14 Anlässlich des 30. Internationalen Orientalistenkongresses von 1976 (deren erster 1873 in Paris stattgefunden hatte) war die UNESCO zu keiner weiteren finanziellen Unterstützung des Kongresses bereit, wenn die Konferenz sich nicht in „International Conference of Asian and North African Studies" (ICANAS) umbenennen würde. Die Forderung wurde erfüllt. Im September 2007 fand übrigens in Ankara die 38. ICANAS-Konferenz statt.

15 Daneben existieren zurückhaltendere Bezeichnungen, wie etwa „Seminar für Sprachen und Kulturen des Vorderen Orients“ (Heidelberg) oder „Seminar für Orientalistik“ (Kiel und Marburg). Eine Umbenennung der Seminare ist nicht leicht möglich, denn es ist eben ,der Orient", der die in diesen Seminaren vertretenen Fächer (wie auch immer) verbindet.

16 Siehe den altbekannten - schon bei Said angelegten - Widerspruch in den Polemiken gegen die Orientalistik bzw. den Orientalismus: Einerseits schrumpft die antiquierte Orientalistik, eine der wesentlichen Trägerinnen des Orientalismus, in ihrem Geltungsbereich und verliert zusehends an Ansehen, andererseits ist aber der Orien- 
Islamwissenschaft und Orientalistik teilen die Auffassung, dass sie letztlich in der Philologie verankert sind, und verteidigen die ,philologische Primärmethode“"17 als ihr grundlegendes Kapital. Eugen Wirths Feststellung aus den 1970er Jahren, die Orientalistik sei konzentrisch angeordnet ,vom Allerheiligsten (Philologie, Literaturwissenschaft und Beschäftigung mit den schriftlichen Quellen) hin zu den Rändern der Orientalistik mit Feldern wie Sozial- und Wirtschaftsgeschichte" hatte und hat wohl auch immer noch für die Islamwissenschaft Gültigkeit. ${ }^{18}$ Der Grund für die so nachhaltige Bindung an die Philologie, und zwar im Sinne einer über die pure Sachnützlichkeit hinausgehenden emotionalen Verpflichtung, ${ }^{19}$ muss auch in der Erfahrung der Orientalistik liegen, sich unter Berufung auf ihre philologische Kompetenz erfolgreich von übergeordneten Fachtraditionen losgelöst zu haben. Der Orientalistik war es (mitsamt der damals als eigenes Fach noch nicht existierenden Islamwissenschaft) im frühen 19. Jahrhundert gelungen, sich von ihrer Rolle als Magd der Theologie zu emanzipieren, und zwar dadurch, dass sie sich auch als Expertin für lebende orientalische Sprachen zu empfehlen begann.

Wenn in dieser Geschichte der Emanzipation ein Grund für die starke Bindung an die Philologie liegt, so ist darin auch der Keim der Selbstüberschätzung enthalten: Die Philologie wird mitunter zu einer Art Totem erhoben: Ende der 1990er Jahre führte der damalige Vorsitzende der Deutschen Morgenländischen Gesellschaft die Erfolglosigkeit der deutschen Entwicklungshilfe auf den falschen Ansatz zurück, zur Entwicklung fremder Kulturen beizutragen zu wollen, ohne von ihnen eine genauere innere Kenntnis zu besitzen. Deswegen seien Forschungszentren einzurichten, ,in denen auf streng philologischer Grundlage die Besonderheit der fremden Kultur und ihrer inhärenten Wertmaßstäbe Gegenstand der Forschung sein müßte. ${ }^{20}$ Man darf vermuten, dass diese Ermahnung bei dem

talismus weiterhin so wirkungsmächtig, dass er nach wie vor den orientalischen und insbesondere den muslimischen Anderen problemlos fehl-repräsentieren und fehlkonstruieren kann. Ich verdanke diesen wichtigen Hinweis Manfred Sing.

17 Walter Slaje: „Was ist und welchem Zweck dient Indologie? Tractatus irae“, in: ZDMG 153 (2003), S. 311-331, hier S. 321: „Die philologische Primärmethode [Heraushebung im Original] aber schließt uns die geistige Welt dieser Zivilisation als dafür notwendige Grundlage zunächst über ihre sprachlichen Dokumente - von den chiffriert überlieferten Gedanken her - auf.“

18 Eugen Wirth: „Orientalistik und Orientforschung. Aufgaben und Probleme aus der Sicht der Nachbarwissenschaften“, in: W. Voigt (Hg.), XIX. Deutscher Orientalistentag, S. LVII.

19 Auffallend ist immer wieder der beschwörende Ton, wenn an die Unbedingtheit der philologischen Grundlagen erinnert wird. Siehe als ein Beispiel die Schlusssätze in Josef van Ess: „The Emergence of ,Kulturgeschichte“ in Islamic Studies“, in: Malcolm H. Kerr (Hg.), Islamic Studies: A Tradition and its Problems, Malibu: Undena Publications 1980, S. 27-51, hier S. 51: „There is no valid historical research withouth philology. We need Herder as well as Hegel.“

20 Eröffnungsansprache des Ersten Vorsitzenden der Deutschen Morgenländischen Gesellschaft, Hermann Jungraithmayr, am 28.9.1998, in: Stefan Wild/Hartmut 
damaligen Bundesminister für Finanzen und der damaligen Bundesministerin für Zusammenarbeit und Entwicklung nicht zu tiefer innerer Einkehr geführt haben wird.

In ihrer öffentlichen Sichtbarkeit und Repräsentanz in den Universitäten unterscheiden sich allerdings die orientalistischen Fächer heute erheblich. ${ }^{21}$ Fächer wie Indologie und Semitistik, die eine starke Forschungstradition in Deutschland aufweisen, aber in der derzeitigen Wahrnehmung der Wissenschaftsverwaltungen als antiquiert gelten, ${ }^{22}$ werden abgebaut, währenddessen andere Fächer wie die Islamwissenschaft oder Sinologie angesichts der Bedeutung, die den entsprechenden Regionen (islamische Welt bzw. China) zugesprochen wird, in ihrem Bestand belassen werden. Die Sinologie darf als ,Wirtschaftsgewinnlerin ' bezeichnet werden, während die Islamwissenschaft wenn nicht als ,Kriegsgewinnlerin“, so doch zumindest als ,Konfliktgewinnlerin“ gelten muss.

Die zunehmende Antiquiertheit des Begriffes und der gemeinsamen Fächertradition ,Orientalistik' ist den orientalistischen Fächern selbst bekannt. Eine der möglichen Antworten wäre, sich von dieser Tradition zu distanzieren bzw. sich schlicht von ihr loszusagen. So haben sich aus den letzten Orientalistentagen (dem etwa alle drei Jahre stattfindenden Fachkongress der deutsprachigen Orientalistik) einzelne Fächer wie die Ägyptologie vollständig und andere wie die Afrikanistik, Indogermanistik, Japanologie, Judaistik und Südostasienkunde weitgehend verabschiedet. $^{23}$

Jedoch würde es nicht gelingen und wohl auch schädlich für einzelne Fächer sein, die Orientalistik kurzerhand für abgeschafft zu erklären. Denn aufgelöst

Schild (Hg.), Norm und Abweichung. Akten des 27. Deutschen Orientalistentages (Bonn, 28. September bis 2. Oktober 1998), Würzburg: Ergon 2001, S. 3-9, hier S. 5.

21 Zum Kanon der Orientalistik gehören die Fächer Ägyptologie, Afrikanistik, Alter Orient, Arabistik, Christlicher Orient/Byzanz, Indogermanistik, Indologie, Iranistik, Islamwissenschaft, Japanologie, Judaistik, Kunst und Archäologie des Orients, Moderner Orient, Orientalische Kunstgeschichte, Orientgeographie, Semitistik, Sinologie, Südostasienkunde, Turkologie/Osmanistik, Zentralasienkunde. Dieser Fächerkanon ist übrigens an keiner Stelle autoritativ festgelegt. In ihren verschiedenen Satzungen legt die Deutsche Morgenländische Gesellschaft als ihr Ziel die Erforschung des Morgenlandes fest, ohne dieses näher zu beschreiben; siehe z.B. die Satzung vom Oktober 1903: „Die Kenntnis des Morgenlandes (im weitesten Sinne)“, in: ZDMG 67 (1913), S. LXXI.

22 Man kann davon ausgehen, dass sich die Haltung gegenüber der Indologie in den nächsten Jahren rasch verändern wird; fraglich hingegen ist, ob die Semitistik, obwohl noch vor wenigen Jahrzehnten nahezu ein deutsches Wissenschaftsoligopol, mehr Anteilnahme in den Wissenschaftsverwaltungen finden wird.

23 Als Hinweis hierfür können die Veranstaltungsprogramme der Orientalistentage in den Jahren 2001 (web.uni-bamberg.de/split/dot), 2004 (www.dot2004.de) und 2007 (www.dot2007.de) dienen. Diese und der Orientalistentag von 1998 in Bonn wurden von Islamwissenschaftlern organisiert und waren dementsprechend von deren Interessen geprägt. 
würden mit den Orientalischen Seminaren in erster Linie die Fächer und nicht ,der Orient", auf den vor allem auch die Nicht-Orientalistik nur schwer wird verzichten wollen. Der Orient, auch wenn er von der Liste der korrekten wissenschaftlichen Begrifflichkeiten getilgt worden sein mag, macht sich als unterdrücktes Unbewusstes immer wieder geltend.

Was also könnte heute die Aufgabe der Orientalistik sein? Ist sie ein Konglomerat von regional- bzw. kulturwissenschaftlich orientierten Fächern mit einer stark philologischen Prägung? Sollte sich eine wissenschaftliche Organisation wie die Deutsche Morgenländische Gesellschaft vor allem als Vereinigung verstehen, die die gemeinsamen Interessen ,kleiner' kulturwissenschaftlich ausgerichteter Fächer an deutschen Universitäten vertritt? Darüber hinaus gehend aber stellt sich die Frage: Welches Anliegen können - über die gemeinsame Herkunft und über das gemeinsame wissenschaftspolitische Anliegen in der deutschen Universitätslandschaft hinausgehend - diese Fächer miteinander teilen? Die Orientalistik spiegelt nach wie vor die Vielfalt der darin vertretenen Disziplinen und Philologien wider; sie scheint aber nicht fähig, eine Auseinandersetzung der orientalistischen Disziplinen (aber auch innerhalb der einzelnen Disziplinen) ${ }^{24}$ darüber zustande zu bringen, in welcher Weise Orientalistik noch ein sinnvoller Wissenschaftsverbund sein könnte. ${ }^{25}$ Es ist auch bezeichnend, dass nicht die Orientalistik, sondern die Geschichtswissenschaft als ,zankende Zunft“ tituliert wurde. ${ }^{26}$ In dieser Bezeichnung ist auch eine gewisse Anerkennung für die lebendige Streitkultur in der Geschichtswissenschaft enthalten: Offensichtlich haben weder Historikerstreit noch Historikerstreitigkeiten dem Fach Geschichte geschadet, sondern seinen Stand in der Öffentlichkeit eher befestigt.

Ohnehin schrumpft der Geltungsbereich der Orientalistik oder das, was als Orientalistik verstanden wird. Den meisten Außenstehenden käme es heute nicht

24 Hans-Wilm Schütte: Die Asienwissenschaften in Deutschland. Geschichte, Stand und Perspektiven, Hamburg: IFA 2002, S. 251; zur fehlenden Suche der Indologie nach einer Verständigung über das Wesen ihres Faches: „Aber haben sich die Indologen je zu Tagungsserien getroffen, um ihr Selbstverständnis zu diskutieren? Nahmen entsprechende Debatten auf den deutschen Orientalistentagen breiten Raum ein? Davon ist nichts bekannt."

25 Symptomatisch für ,Glanz und Elend " der Orientalistik ist die Zeitschrift der Deutschen Morgenländischen Gesellschaft, die für Beiträge aus allen orientalistischen Disziplinen ein höchst bewährtes Publikationsforum bietet, aber zugleich ernüchternd ist in der Zusammenhangs- und Kommunikationslosigkeit der unterschiedlichen Beiträge. Siehe eine ähnliche Einschätzung der Problematik bereits bei Carl Brockelmann: „Die morgenländischen Studien in Deutschland“, in: ZDMG 76 (1922), S. 1-17, hier S. 14: „So konnte es schließlich nicht ausbleiben, daß unsere Zeitschrift, da sie im Gegensatz zu den Fachorganen auf zufällig ihr zufließende Beiträge aus allen Gebieten der Orientforschung angewiesen blieb, jeden organischen Aufbau vermissen ließ.“

26 Klaus Große Kracht: Die zankende Zunft. Historische Kontroversen in Deutschland nach 1945, Göttingen: Vandenhoeck \& Ruprecht 2005. 
mehr in den Sinn, die Sinologie als genuin orientalistisches Fach zu verstehen. An manchen Standorten findet sich noch die Sinologie im Verbund von Orientalischen Seminaren, aber die Islamwissenschaft ist meist das dominierende, manchmal sogar das einzige darin vertretene Fach - vor allem, wenn man nicht nur die Disziplin Islamwissenschaft selbst berücksichtigt, sondern auch zahlreiche weitere, der Islamwissenschaft nahe stehende Fächer wie Turkologie, Arabistik und Iranistik, die sich mit den jeweils behandelten Kulturen in ihrer islamischen Überprägung auseinandersetzen. Man möchte also vermuten, dass die heute stärkste und weithin unangefochtene Spielart der Orientalistik die Islamwissenschaft ist. Gerade die letzten Jahre vermitteln auch den Eindruck, als sei der Islam in der Wahrnehmung der westlichen Öffentlichkeit anstelle des Orients in den Part des grundsätzlich Anderen eingetreten. Wenn man also die Existenz eines ,harten Kern Orients“ heute noch vermuten will, dann muss es sich wohl um den Islam handeln. Diese Erwartung der Öffentlichkeit ist höchst bedenklich auch für die Islamwissenschaft.

\section{Erst Überforderung und dann Marginalisierung?}

„Vor zwei oder drei Generationen war der Anspruch der Orientalisten, kraft ihrer philologischen Fähigkeiten exklusiv für die Erkenntnis, Interpretation und Vermittlung zuständig zu sein, gesellschaftlich anerkannt und garantiert. In diesem Bewusstsein verwalteten wir Generationen hindurch das Exotische, Außergewöhnliche und Fremde und konnten uns dank unserer bisweilen zu höchsten Höhen der Kunst zugespitzten philologischen Kenntnisse auch der allgemeinen Wertschätzung sicher sein. Fragen nach jeweils zeitgenössischen, gegenwärtigen Verhältnissen setzten wir oft erfolgreich den höherrangigen Wert des Alten, des Historischen, des Klassischen entgegen und waren uns der Akzeptanz derartiger Thesen sicher. “27

Ein solches Verharren in den olympischen Höhen der Orientalistik ist für die Islamwissenschaft längst nicht mehr möglich. Wiederholt wurde an der Islamwissenschaft als Fach bemängelt, dass sie sich nicht als Gesprächspartner der Öffentlichkeit und der Politik bewiesen hat. Es scheint aber derzeit trotzdem so, als wolle man angesichts des kulturalistisch und identitätspolitisch geprägten Konflikts mit der islamischen Welt die Islamwissenschaft noch einmal zur Bewährung in den Rang eines Generalbevollmächtigten für das Andere einsetzen. Aber wird ihr dieser Status wegen erwiesener Inkompetenz und Untätigkeit nicht bald wieder entzogen werden? Dieser Überforderung nicht gerecht zu werden und da-

27 Ansprache des Ersten Vorsitzenden der Deutschen Morgenländischen Gesellschaft, Bert Fragner, bei der Eröffnung des XXVIII. Deutschen Orientalistentags, 26.-30. März 2001: „Orientalistik zwischen Philologie und Sozialwissenschaft“, http://web. uni-bamberg.de/split/dot/reden_fragner vom 5.4.2007. 
für die Strafe der Marginalisierung gewärtigen zu haben, mit dieser Sachlage steht die deutsche Islamwissenschaft nicht alleine da. So konnten die zahlreichen think tanks in den USA in der öffentlichen Meinungsbildung die Vertreter der Universitäten vor allem deswegen an den Rand drängen, weil sie eher dazu fähig waren, Sachverhalte verständlich und in Kürze darzustellen, aber auch das ,nati-

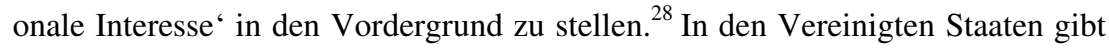
es ohnehin eine längere Tradition der unmittelbaren Einbindung von akademischen Ressourcen für sicherheitsdienstliche Belange, etwa in Gestalt des ,Camelot'-Programms (eines Vorhabens der US-amerikanischen Armee in den 1960er Jahren), nach dem die in den US-amerikanischen Universitäten verankerten Area Studies, unter anderen auch die für den Nahen Osten, für die Entwicklung einer effektiven Politik der counter insurgency herangezogen werden sollten. ${ }^{29}$

Auch wenn bisher solche unmittelbar sicherheitsrelevanten Erwartungen nicht an die deutsche Islamwissenschaft herangetragen worden sind (wohl weniger aus wissenschaftlicher Pietät als aus der Überzeugung, dass dies ohnehin keinen Sinn hätte), so scheint sie auch nicht dazu in der Lage zu sein, allgemeine Erklärungsmodelle zum ,Problemfall Islam‘ anbieten zu können. ${ }^{30}$ Mittelfristig steht die Islamwissenschaft in Gefahr, von den Mühlen einer an sie herangetragenen übermäßigen Erwartung und einer (aus dieser enttäuschten Erwartung heraus begründeten) Geringschätzung und Marginalisierung zermahlen zu werden.

Die Islamwissenschaft sieht sich dem grundsätzlichem Problem gegenüber, dass ihre Selbstbezeichnung Erwartungen weckt, die sie nicht einlösen kann. Es geht hier weniger um die im Wort ,Islamwissenschaft" enthaltene Ankündigung, zur Gesamtheit der islamischen Welt in historischer Tiefe und geographischer Breite Auskunft geben zu können. Weitaus schwerer wiegt die Erwartung der Öffentlichkeit, dass die Islamwissenschaft den unauflösbaren, nicht zu greifenden Kern ,Islam‘ erklären soll: Die Islamwissenschaft soll sagen, worin das Geheimnis des eigentlichen Orients, der heute der Islam ist, liegt, aber eben nicht, um sich einem fremden kulturellen Mysterium zu nähern, sondern um zu erläutern, wie der Muslim als solcher ,tickt‘. ${ }^{31}$

28 Martin Kramer: Ivory Towers on Sand: The Failure of Middle Eastern Studies in America, Washington, D.C.: The Washington Institute for Near Eastern Policy 2001, S. 106f.

29 Ebd., S. 86; zu späteren Vorstößen von Regierungsorganisationen siehe S. 87-90.

30 Siehe hierzu auch die Anmerkung von Jacques Waardenburg: „The Study of Islam in German Scholarship“, in: Azim Nanji (Hg.), Mapping Islamic Studies. Genealogy, Continuity and Change, Berlin, New York: Mouton de Gruyter 1997, S. 1-32, hier S. 21: „The situation is the more astonishing since German culture has always had a speculative bent and German scholarship is reputed for its creative theoretical thought. Why precisely this German genius has been largely absent in the field of Islamic studies is a problem."

31 Dass auch Bomben ticken, ist hier ein nicht ganz zufälliges Zusammentreffen des Wortsinns. 
Aus ihrer philologischen Tradition heraus ist die Islamwissenschaft stark, wenn es um die Beschreibung von Normativitäten geht, die sie aus der Lektüre von Texten gewinnt. Texte, sakrale Texte vor allem, legen auch - so der eigene Anspruch der Muslime - religiöses Verhalten fest. Das ist nicht ohne Fallstricke: Weil Muslime in der Weise wahrgenommen werden, dass sie die religiösen und dogmatischen Inhalte des Islams mit größerem Nachdruck als säkularisierte Christen verfechten, wird von ihnen ein solches streng religiöses Verhalten dann auch als zwingend vorausgesetzt. Die deutsche Öffentlichkeit, so ließe sich überspitzt ausdrücken, erwartet von den Muslimen ,,eben diese Übereinstimmung [ihres] Verhaltens mit ihren eigenen Normen “32 und von der Islamwissenschaft erwartet sie nur noch die Bestätigung dieser Erwartungen. Den als Muslimen erzogenen Menschen wird ihre religiöse Identität geradezu aufgedrängt - und den Islamwissenschaftlern ihre angebliche Identität als Bewahrer des Schlüssels zum eigentlichen Verständnis des Islams.

Das Dilemma der Islamwissenschaft ist, dass viele Vertreter dieser Disziplin selbst der Ansicht sind, bei den meisten politischen Entwicklungen unter Muslimen und in der islamischen Welt handle sich nicht um nur dem Islam zueigene Charakteristika, sondern viel eher um bestimmte gesellschaftliche Strukturen oder der um spezifische Antworten auf die Herausforderung der Moderne. Die islamische Welt, enttäuscht von falschen europäischen Versprechungen wie dem Liberalismus und Marxismus, versuche, die Moderne durch eine islamisierende Überformung für sich verstehbar und bewältigbar zu machen. Auf die Spitze getrieben bedeutet dies, dass die Islamwissenschaft sich gewissermaßen als nicht zuständig erklärt für alles Islamische, da es ja nur scheinbar islamisch sei. Worin könnte also der Sinn eines Faches liegen, das - so stellt sich das dem Außenstehenden dar - sich vor allem berufen sieht, gerade das als irrelevant zu bezeichnen, was es erklären soll?

Hierin könnte auch einer der Gründe dafür liegen, warum die Islamwissenschaft in der Öffentlichkeit als so stumm erscheint. Die Islamwissenschaft weiß vielleicht, was man nicht sagen kann, indem sie das Einspruchsrecht der Fakten kennt. Sie weiß aber nicht, was man sagen kann; dazu müsste sie selbst erst gelernt haben, über Muslime und Islamisches reden zu können, ohne dass diese Rede immer letztlich ,den Islam“ meinen würde.

Das Fach Islamwissenschaft muss sich nicht notwendigerweise selbst beschädigen, wenn es ihm gelingen sollte, im Sinne des von Odo Marquardt auf die Philosopie gemünzten Begriffes der ,Inkompetenzkompensationskompetenz', auf diese Dilemmata hinzuweisen und verständlich zu machen, dass die falschen Erwartungen an die Islamwissenschaft Teil eines größeren Missverständnisses sind.

32 Peter Heine: Kulturknigge für Nichtmuslime. Ein Ratgeber für alle Bereiche des Alltags, Freiburg: Herder 2001, S. 158. 
Worin also könnte die Antwort der Islamwissenschaft auf - jenseits all ihrer unbestrittenen Kompetenzen - ihre spezifische Inkompetenz sein, die Frage „Nun sagen Sie uns doch einmal gute Frau/guter Mann, was ist denn der Islam eigentlich?" nicht befriedigend beantworten zu können oder genauer vielleicht, nicht die Fähigkeit zu haben, diese Frage in überzeugender Weise als unzulässig abzuwehren.

\section{Was kann die Islamwissenschaft tun?}

„Aus den Stürmen der kurz zuvor über ganz Europa hereingebrochenen politischsocialen Bewegung, die auch mein Vaterland so mächtig ergriffen hatte, sah ich mich plötzlich in die durch nichts gemilderte Einförmigkeit des orientalischen Lebens versetzt. Während ich daheim alles hatte wanken sehen, stand hier alles unerschütterlich, unbeweglich und jeden Zweifel ausschliessend. Nichts störte die tiefe Ruhe.“33

Man könnte diese Passage auch als ein Gleichnis auf die Islamwissenschaft der ersten Nachkriegsjahrzehnte lesen, als sie noch weitgehend unberührt von innenund außenpolitischen Stürmen in sich und ihren Traditionen ruhte. In den gemütlichen islamwissenschaftlichen Seminaren waren deutsche Studierende und Dozierende unter sich; gemeinsam sprach man über den zeitlich und räumlich so fernen Islam. Mit dieser Gemütlichkeit scheint es erst einmal vorbei zu sein.

Dabei wäre eigentlich das Streben nach einer akademischen vita contemplati$v a,{ }^{34}$ vulgo das Pochen auf Ruhe als oberstem Wissenschaftlerrecht, nicht nur eine wohlfeile Ausrede - dies zeigen Plädoyers zur Rettung der deutschen Universität, in denen die Forderung nach Kontemplativität an oberster Stelle steht: „Was die Universität heute braucht, ist Ruhe.“35

Dennoch: Die Islamwissenschaft wird in den nächsten Jahren nicht - jedenfalls nicht mit Erfolg - das Privileg der Ruhe einfordern können. Viele Herausforderungen warten auf sie, anregende, aber auch einfach unangenehme. Um auf diese Herausforderungen antworten zu können, wird die Islamwissenschaft eine Gratwanderung unternehmen müssen: Sie darf nicht ihre Kompetenzen verlieren und muss zugleich versuchen, spezifische Formen einer islamwissenschaftlichen ,Inkompetenzkompensationskompetenz' zu schaffen.

Manches von dem, was zum Abschluss dieses Beitrags über den möglichen Auftrag der Islamwissenschaft in der Zukunft gesagt wird, ist handhabbar, man-

33 Alfred von Kremer: Geschichte der herrschenden Ideen des Islams. Der Gottesbegriff, die Prophetie und Staatsidee, Hildesheim: Georg Olms 1961. Nachdruck der Ausgabe von 1. Auflage, Leipzig 1868, S. X.

34 L. Richter-Bernburg: Islamwissenschaft, S. 126.

35 Jochen Hörisch: Die ungeliebte Universität. Rettet die Alma mater! München, Wien: Hanser 2006, S. 134. 
ches geht über allerlei Wolkiges nicht hinaus. Beim Blick in die vermeintlich wichtigsten Zukunftsaufgaben der Islamwissenschaft tun sich aber auch intellektuell so bestechende Autoren wie Baber Johansen schwer. Johansen sah es 1990 als eine hauptsächliche Aufgabe der Islamwissenschaft an, sich einem „,neuen Orientalismus" zu widersetzen, der den Nahen Osten nur als politisches Gebilde und als wirtschaftlichen Faktor sehen könne (heute müsste natürlich an erster Stelle die Reduzierung der Muslime und des Islams auf ein Sicherheitsproblem stehen). Um dies leisten zu können, müsse erstens die Islamwissenschaft ihre Tradition bewahren, das Textuniversum der islamischen Welt zu erforschen und verstehen zu wollen, zweitens eine engere Vertrautheit mit den Sprachen und Traditionen ihrer eigenen (also der europäischen) Kultur und ihrer Abbildung in den verschiedenen akademischen Traditionen erwerben, und drittens ein weitaus sensibleres Sensorium für die kulturellen Strömungen und emotionalen Zustände in der islamischen Welt entwickeln. Darüber hinaus plädiert er dafür, dass die Islamwissenschaft für die Zwecke ihrer Analysen einen ,theoretischen, empirischen und historischen Bezugsrahmen" entwickeln solle. ${ }^{36}$ Es scheint wohl so, als ob niemand der Islamwissenschaft einen klaren Weg in die Zukunft weisen könne.

Die Islamwissenschaft in Deutschland ist in den letzten Jahrzehnten zu sehr Handwerk gewesen: Philologische Überlastung, unter anderem durch die nur sehr selten wirklich eingelöste Verheißung, mehrere islamische Sprachen (vorzugsweise Arabisch, Persisch und Türkisch) zu erlernen, kann schon während des Studiums zu einem Defizit an intellektueller Ausbildung beitragen. Zudem besteht ein Missverständnis sowohl auf Seiten der Islamwissenschaftler als auch der Außenstehenden: Islamwissenschaftler sollten sich als Intellektuelle zu Fragen äußern können, die den Islam und die Muslime in den Gesellschaften der Gegenwart betreffen, ohne allesamt zu sich mit der unmittelbaren Moderne beschäftigenden Sozialwissenschaftlern mutieren zu müssen. Man kann und sollte über - für andere - entlegene Themen und Dinge forschen und trotzdem dazu fähig sein, sich im öffentlichen Raum zu behaupten.

Die Deutungshoheit der Islamwissenschaft, wenn es jemals eine solche gegeben haben sollte, ist auch durch die Migration von Muslimen nach Europa erodiert worden. Orientalistik als Disziplin setzte ein klar definiertes Eigenes voraus - und dieses klar definierte Eigene war auch im Fall der Islamwissenschaft immer Europa mit seiner spezifisch christlich-abendländischen Kultur. Schon 1983 beklagte der 1938 aus Deutschland emigrierte und in den USA lehrende Islamwissenschaftler Franz Rosenthal (1914-2003), der Orientalistik sei das Fremde abhanden gekommen: Die muslimischen Migranten nach Europa würden „,an den

36 B. Johansen: Politics and Scholarship, S. 115: „Islamic studies ought to have a theoretical, empirical, and historical frame of reference that enables it to analyze cultures in terms of interactions between individuals, groups, and classes $[\ldots]$ ". 
ererbten Grundlagen der Orientalistik rütteln und sie zu zerstören drohen. Es sieht so aus, als ob der Reiz des Fremden nicht mehr existieren könne und die Suche nach dem Eigenen in diesem einst Fremden nicht mehr möglich sei, da das Fremde eben allzu vertraut und alltäglich geworden zu sein scheint.“37

So leicht aber geht das Fremde nicht verloren. Der Begriff ,Muslim“ hat in Europa bereits teilweise eine neoethnische oder pseudoethnische Konnotation angenommen, indem man dem Muslim nicht nur eine religiöse Zugehörigkeit, sondern gewissermaßen eine ethnisch-rassische zuweist. Abhilfe lässt sich womöglich unter anderem durch die Schaffung von Räumen bewerkstelligen, in denen Muslime und Nicht-Muslime eben nicht als Parlamentäre verfeindeter Formationen aufeinandertreffen (was übrigens ein Grundproblem aller ,Dialog ‘Bemühungen ist), ja, in denen solche Zuordnungen einem gar nicht mehr in den Sinn kommen. Unter anderen könnte die Islamwissenschaft Ort eines solchen Austauschs sein, in dem Muslime und Nicht-Muslime eben nicht mehr als Muslime und Nicht-Muslime, sondern als gemeinsam arbeitende Studierende, Lehrende und Forschende aufeinandertreffen. ${ }^{38}$

Notwendig ist auch eine klarere Vermittlung des Faches im Unterricht. Es ist eine Illusion zu glauben, die enorme fachliche Breite der Islamwissenschaft könne in der Lehre eins zu eins wiedergegeben werden. Die Herausforderung für die geisteswissenschaftlichen Fächer, nämlich ,das Studium in eine Sequenz klar bestimmter Schritte so zu zerlegen, dass die Studenten einen Studiengang erleben, in dem Geleistetes aufeinander aufbaut und sich nicht nur impressionistisch zu einem Bild fügt" “ ${ }^{39}$ lässt sich besonders als Aufgabe für die islamwissenschaftlichen Studiengänge verstehen.

Eine weitere Herausforderung für die Islamwissenschaft ist, sich in Zukunft zu einzelnen politischen Entwicklungen und Fragestellungen deutlich zu äußern. Dass Islamwissenschaftler in der deutschen Islamkonferenz kaum vertreten sind, muss man als Schicksal hinnehmen. Manches in der Zukunft Liegende könnte aber noch von der Islamwissenschaft beeinflusst werden: So braucht die Islamwissenschaft nicht Anspruch darauf zu erheben, die Lehrer für den islamischen Religionsunterricht auszubilden, aber sie muss darauf bestehen, dass die Dozenten, die diese Lehrer ausbilden (werden), einen festen Anteil ihrer Ausbildung

37 F. Rosenthal: Krise der Orientalistik, S. 15.

38 Das wird nicht immer einfach sein: Eine der Stärken westlicher Wissenschaft, nämlich wissenschaftliche Kritik, können manche der muslimischen Studierenden nur als Kritik an ihrer eigenen Religion verstehen. - Die Sinologie und die Slavistik mit einer zunehmenden Zahl von chinesischen bzw. russischen Muttersprachlern/innen unter ihren Studierenden kennen in den letzten beiden Jahrzehnten eine ähnliche Entwicklung; im Fall der Islamwissenschaft aber treten die religiös-kulturellen Trennlinien wohl noch deutlicher hervor.

39 Jürgen Kaube: „Das Unbehagen in den Geisteswissenschaften. Empirie und überempirische Krisen“, in: F. Keisinger/R. Berger (Hg.), Wozu Geisteswissenschaften, S. 17-28, hier S. 23. 
nicht nur in Studiengängen der islamischen Theologie erhalten, sondern auch in der Islamwissenschaft. Es geht hier nicht darum, dass Dozenten des islamischen Religionsunterrichts eine distanzierte Sicht auf ihre Religion einüben sollen, sie sollen aber zumindest die Haltung einer kritischen Distanz kennenlernen, die in der islamischen Theologie und Wissenschaftstradition nicht sehr ausgeprägt ist.

Über allem mea culpa soll aber auch nicht das tua culpa vergessen werden. Es ist ein Zeichen für den Orientalismus und Provinzialismus der deutschen Gesellschaft, dass sie erwartet, jeweils eine Handvoll von Fachvertretern könne eine Region in allen ihren Aspekten erklären, die weitaus größer ist als Westeuropa und Nordamerika zusammengenommen. Wie würde gelacht werden, wenn jemand den Vorschlag wagte, in Zukunft die Theologie, Geschichte, Philosophie, Soziologie, Politik-, Religions- und Literaturwissenschaft an einer Universität durch ein, zwei ,Okzidentalisten “ vertreten zu lassen? Es geht hier nicht um Lobbyismus, nämlich dass Gedanken zur Lage der Islamwissenschaft in Deutschland zwangsläufig in der Forderung nach mehr Stellen für die Islamwissenschaft münden müssen, sondern darum, dass an der Erklärung der ungeheuer großen und vielfältigen, islamischen Welt" auch andere, bisher abseits stehende Fächer wie die Geschichtswissenschaft teilhaben sollten. Warum sollte nicht etwa das Fach Geschichte in zumindest einigen Universität dazu in die Lage gebracht werden, eine Generation von auf die Gebiete der islamischen Welt spezialisierten und zugleich historisch arbeitenden Wissenschaftlern aufzunehmen - die schon in der nächsten Generation Historiker und eben nicht Islamwissenschaftler hervorbrächten $?^{40}$

Die Islamwissenschaft wird wegen ihrer ausgeprägten philologischen Orientierung und wegen ihrer nicht leicht zu behebenden Tradition einer, oft eklektizistischen Anleihe bei verschiedensten Disziplinen in absehbarer Zukunft keine für die allgemeinen Geisteswissenschaften wegweisenden Pfade beschreiten. Die Islamwissenschaft kann und soll auch kein Methodenfach werden. Es wäre unfruchtbar zu verlangen, dass jeder Islamwissenschaftler sich in Zukunft nur als Religionswissenschaftler, nur als Soziologe, nur als Literaturwissenschaftler, nur als Historiker, nur als Anthropologe usw. zu verstehen hätte. Das methodisch Chaotische oder - positiv gefasst - der disziplinäre Kosmopolitismus der Islamwissenschaft sind durchaus bewahrenswert. Dennoch ist die Konzentrierung auf ein, höchstens aber zwei methodische Schwerpunkte und sehr wenige inhaltliche

40 Die bisherige Bilanz (auch wenn in dieser Übersicht ein, zwei Professuren übersehen worden sein mögen) lässt eher vermuten, dass die eurozentrischen Beharrungskräfte der deutschen Geschichtswissenschaft die Oberhand behalten werden. Bisher gab es im Fach Geschichte an deutschen Universitäten nur folgende Professuren mit einer auf die islamische Welt bzw. den Nahen Osten bezogenen Ausrichtung: Helmut Mejcher in Hamburg (keine Nachfolge), Dan Diner in Essen (beim Nachfolger ersetzt durch einen anderen Schwerpunkt), Fikret Adanır in Bochum (Nachfolge noch unklar), Birgit Schäbler (Erfurt), Alexander Schölch, später Thomas Philipp in Erlangen (Nachfolge noch unklar). 
Felder notwendig. Nur so kann die Islamwissenschaft im besten Sinne eine Disziplin von ,Universal-Spezialisten“ bleiben. ${ }^{41}$ Das Fach kann jedoch versuchen, aus dem Gespräch mit anderen Disziplinen und dem intensiven Nachdenken über das eigene Fach eigenständig Fragestellungen entwickeln - also ein Fach mit einem spezifischen Gewicht und Gehalt sein. Noch zu oft argumentiert die Islamwissenschaft mit dem Hinweis auf ein Arkanum Islamwissenschaft, das sich nur dem mitteilbar machen könne, der selbst auch Islamwissenschaft studiert habe.

Es gilt, dass sowohl klassische Islamwissenschaft als auch Gegenwartsbezogenheit in der Islamwissenschaft ihren Platz haben. Auch ist das Fach nicht dazu da, ,kurzfristig verwertbare, pragmatische Ergebnisse oder gar nur Entscheidungshilfen für Politik und Wirtschaft zu liefern“. ${ }^{42}$ Das Fach hat das Recht, auf seinen Wissenschaftstraditionen zu beharren, auf seiner ihm eigenen Art der ,relevanten Redundanz“. ${ }^{43}$ Die Islamwissenschaft muss sich aber zugleich der Ungeduld stellen, die ihr gegenüber gezeigt wird. ,Aristokratische" Selbstrechtfertigungen, dass man sich eigentlich nicht rechtfertigen und nicht zeitgemäß sein müsse, gehören zur unverzichtbaren Folklore der Universität und sind für das Überleben der Universität vielleicht sogar unverzichtbar - sofern sie allerdings produktive Wirkung entfalten. Die Islamwissenschaft wird bestehen können, wenn sie ihren Wert für andere, aber vor allem für sich selbst erweist.

41 H.-W. Schütte: Asienwissenschaften, S. 292, bezieht sich hier auf das Fach Sinologie.

42 Das Zitat bezieht sich im Originalkontext nicht auf die Islamwissenschaft, sondern auf die Orientalistik insgesamt; Lother Ledderose: „Begrüssungsansprache“, in Werner Diem/Abdoldjavad Falaturi (Hg.), XXIV. Deutscher Orientalistentag, vom 26. bis 30. September 1988 in Köln. Ausgewählte Vorträge, Stuttgart: Steiner 1990, S. 1-6, hier S. 6.

43 Siehe zum Begriff der ,relevanten Redundanz die Einleitung zum Sammelband. 
Bereitgestellt von | Universitaetsbibliothek Basel

Angemeldet

Heruntergeladen am | 19.09.18 11:24 